Pacific Journal of Mathematic 


\title{
THE GENERALIZED SIMPLEX METHOD FOR MINIMIZING A LINEAR FORM UNDER LINEAR INEQUALITY RESTRAINTS
}

\author{
George B. Dantzig, Alex Orden, Philip Wolfe
}

1. Background and summary. The determination of "optimum" solutions of systems of linear inequalities is assuming increasing importance as a tool for mathematical analysis of certain problems in economics, logistics, and the theory of games $[1 ; 5]$. The solution of large systems is becoming more feasible with the advent of high-speed digital computers; however, as in the related problem of inversion of large matrices, there are difficulties which remain to be resolved connected with rank. This paper develops a theory for avoiding assumptions regarding rank of underlying matrices which has import in applications where little or nothing is known about the rank of the linear inequality system under consideration.

The simplex procedure is a finite iterative method which deals with problems involving linear inequalities in a manner closely analogous to the solution of linear equations or matrix inversion by Gaussian elimination. Like the latter it is useful in proving fundamental theorems on linear algebraic systems. For example, one form of the fundamental duality theorem associated with linear inequalities is easily shown as a direct consequence of solving the main problem. Other forms can be obtained by trivial manipulations (for a fuller discussion of these interrelations, see [13]); in particular, the duality theorem [8; $10 ; 11 ; 12]$ leads directly to the Minmax theorem for zero-sum two-person games [1d] and to a computational method (pointed out informally by Herman Rubin and demonstrated by Robert Dorfman [1a]) which simultaneously yields optimal strategies for both players and also the value of the game.

The term "simplex" evolved from an early geometrical version in which (like in game theory) the variables were nonnegative and summed to unity. In that formulation a class of "solutions" was considered which lay in a simplex.

The generalized method given here was outlined earlier by the first of the authors (Dantzig) in a short footnote [1b] and then discussed somewhat more fully at the Symposium of Linear Inequalities in 1951. Its purpose, as we have

Received August 29, 1953. Copyrighted 1953, by the RAND Corporation and reproduced here by permission of the copyright holder.

Pacific J. Math. 5 (1955), 183- 195 
already indicated, is to remove the restrictive assumptions regarding the rank of the matrix of coefficients and constant elements without which a condition called "degeneracy" can occur.

Under degeneracy it is possible for the value of the solution to remain unchanged from one iteration to the next under the original simplex method. This causes the proof that no basis can be repeated to break down. In fact, for certain examples Alan Hoffman [14] and one of the authors (Wolfe) have shown that it was possible to repeat the basis and thus cycle forever with the value of the solution remaining unchanged and greater than the desired minimum. On the other hand, it is interesting to note that while most problems that arise from practical sources (in the authors' experience) have been degenerate, none have ever cycled [9].

The essential scheme for avoiding the assumptions on rank is to replace the original problem by a "perturbation" that satisfies these conditions. That such perturbations exist is, of course, intuitively evident; but the question remained to show how to make the perturbation in a simple way. For the special case of the transportation problem a simple method of producing a perturbation is found in [1c]. The second of the authors (Orden) has considered several types of perturbations for the general case. A. Charnes has extensively investigated this approach and his writing represents probably the best available published material in this regard $[2 ; 3 ; 4]$.

It was noticed early in the development of these methods that the limit concept in which a set of perturbations tends in the limit to one of the solutions of the original problem was not essential to the proof. Accordingly, the third author (Wolfe) considered a purely algebraic approach which imbeds the original problem as a component of a generalized matrix problem and replaces the original nonnegative real variables by lexicographically ordered vectors. Because this approach gives a simple presentation of the theory, we adopt it here.

2. The generalized simplex method. As is well known, a system of linear inequalities by trivial substitution and augmentation of the variables can be replaced by an equivalent system of linear equations in nonnegative variables; hence, with no loss of generality, we shall consider the basic problem in the latter form throughout this paper. One may easily associate with such a system another system in which the constant terms are replaced by l-component constant row vectors and the real variables are replaced by real $l$-component variable row vectors. In the original system the real variables are nonnegative; in the generalized system we shall mean by a vector variable $\bar{x}>0$ (in the lexicographic sense) that it has some nonzero components, the first of which is positive, 
and by $\bar{x}>\bar{y}$ that $\bar{x}-\bar{y}>0$. It is easy to see that the first components of the vector variables of the generalized system satisfy a linear system in nonnegative variables in which the constant terms are the first components of the constant vectors.

Let $P=\left[P_{0}, P_{1}, \cdots, P_{n}\right]$ be a given matrix whose $j$ th column, $P_{j}$, is a vector of $(m+1)$ components. Let $M$ be a fixed matrix of rank $m+1$ consisting of $m+1 l$-component row vectors. The generalized matrix problem is concerned with finding a matrix $\widetilde{X}$ satisfying

$$
P \widetilde{X}=\sum_{0}^{n} P_{j} \overline{x_{j}}=M
$$

where $\bar{x}_{j}$ (the $j$ th row of $\tilde{X}$ ) is a row vector of $l$-components satisfying the conditions, in the lexicographic sense,

$$
\bar{x}_{j} \geq 0 \quad(j=1,2, \cdots, n),
$$

where the relationship between max $\bar{x}_{0}$ and the minimization of a linear form will be developed in $\S 3$.

Any set $X$ of "variables" $\left(\bar{x}_{0} ; \bar{x}_{1}, \bar{x}_{2}, \ldots, \bar{x}_{n}\right)$ satisfying $(1)$ and $(2)$ in the foregoing lexicographic sense will be referred to as a "feasible solution" (or more simply as a "solution") - a term derived from practical applications in which such a solution represents a situation which is physically realizable but not necessarily optimal. The first variable, $\bar{x}_{0}$, which will be called the "value" of the solution, is to be maximized; it is not constrained like the others to be nonnegative. In certain applications (as in $\$ 3$ ) it may happen that some of the other variables also are not restricted to be nonnegative. This leads to a slight variation in the method (see the discussion following Theorem 5 ).

Among the class of feasible solutions, the simplex method is particularly concerned with those called "basic." These have the properties, which we mention in passing, (a) that whenever any solution exists a basic solution also exists (Theorem 8 ), and (b) that whenever a maximizing solution exists and is unique it is basic solution, and whenever a maximizing solution is not unique there is a basic solution that has the same maximizing value (Theorem 6). A basic solution is one in which only $m+1$ variables (including $\bar{x}_{0}$ ) are considered in (1), the remainder being set equal to zero; that is, it is of the form

$$
B V=P_{0} \bar{v}_{0}+\sum_{i=1}^{m} P_{j_{i}} \bar{v}_{i}=M
$$

$$
\left(\bar{v}_{i} \geq 0, j_{i} \neq 0\right) \text {, }
$$


where $B=\left[P_{0}, P_{j_{1}}, \cdots, P_{j_{m}}\right]$ is an $(m+1)$-rowed square matrix and $V$ is a matrix of $m+1$ rows and $l$-columns whose $i$ th row is denoted by $\bar{v}_{i}(i=0,1, \cdots, m)$.

It is clear from (4) that since $M$ is of rank $m+1$ so are $B$ and $V$. From this it readily follows that the $m+1$ columns of $B$ constitute a basis in the space of vectors $P_{j}$, and the solution $V$ is uniquely determined. Moreover, since the rank of $V$ is $m+1$, none of the $m+1$ rows of $V$ can vanish; that is, it is not possible that $\bar{v}_{i}=0$. Thus in a basic solution all variables associated with the vectors in the basis (except possibly $\bar{v}_{0}$ ) are positive; all others are zero. The condition in (4) can now be strengthened to strict inequality

$$
\bar{v}_{i}>0 \quad(i=1, \cdots, m) .
$$

Let $\beta_{i}$ denote the $i$ th row of $B$ inverse:

$$
B^{-1}=\left[P_{0}, P_{j_{1}}, P_{j_{2}}, \cdots, P_{j_{m}}\right]^{-1}=\left[\beta_{0}^{\prime}, \beta_{1}^{\prime}, \cdots, \beta_{m}^{\prime}\right]^{\prime}
$$

where primed letters stand for transpose.

THE OREM 1. A necessary and sufficient condition that a basic solution be a maximizing solution is

$$
\beta_{0} P_{j} \geq 0 \quad(j=1, \cdots, n) .
$$

THEOREM 2. If a basic solution is optimal, then any other solution (basic or not) with the property that $\bar{x}_{j}=0$ whenever $\left(\beta_{0} P_{j}\right)>0$ is also optimal; any solution with $\bar{x}_{j}>0$ for some $\left(\beta_{0} P_{j}\right)>0$ is not optimal.

Proofs. Let $\tilde{X}$ represent any solution of (1), and $V$ a basic solution with basis $B$; then multiplying both $(1)$ and (4) through by $\beta_{0}$ and equating, one obtains, after noting from (6) that $\beta_{0} P_{0}=1$ and $\beta_{0} P_{i}=0$,

$$
\bar{x}_{0}+\sum_{1}^{n}\left(\beta_{0} P_{j}\right) \bar{x}_{j}=\bar{v}_{0}
$$

whence, assuming $\beta_{0} P_{j} \geq 0$, one obtains $\bar{x}_{0} \leq \bar{v}_{0}$ (which establishes the sufficiency of Theorem 1); moreover the condition $\bar{x}_{j}=0$ whenever $\beta_{0} P_{j}>0$ $(j \neq 0)$ implies the summation term of $(8)$ vanishes and $\bar{x}_{0}=\bar{v}_{0}$; whereas denial of this condition implies the summation term is positive if Theorem 1 is true (establishing Theorem 2). 
In order to establish the necessity of ( 7) for Theorem 1 , let $Y_{s}$ be a column vector which expresses a vector $P_{s}$ as a linear combination of the vectors in the basis:

$$
P_{s}=B\left(B^{-1} P_{s}\right)=B Y_{s}=\sum_{i=0}^{m} P_{j_{i}} y_{i s} \quad\left(P_{0}=P_{j_{0}}\right)
$$

where it is evident from (6) that, by definition,

$$
y_{i s}=\beta_{i} P_{s} \quad(i=0,1, \cdots, m) .
$$

Consider a class of solutions which may be formed from (4) and (9), of the form

$$
B\left[V-Y_{s} \bar{\theta}\right]+P_{s} \bar{\theta}=M,
$$

or more explicitly

$$
P_{0}\left[\bar{v}_{0}-y_{0 s} \bar{\theta}\right]+\sum_{i=1}^{m} P_{j_{i}}\left[\bar{v}_{i}-y_{i s} \bar{\theta}\right]+P_{s} \bar{\theta}=M
$$

It is clear that, since $\bar{v}_{i}>0$ for $i \geq 1$ has been established earlier ( see ( 5$)$ ), a class of solutions with $\bar{\theta}>0$ (that is, with $\bar{\theta}$ strictly positive) always exists such that the variables associated with $P_{s}$ and $P_{j}$ in (12) are nonnegative, hence admissible as a solution of (1). If $y_{0 s}<0$, then the values of these solutions are

$$
\bar{v}_{0}-y_{0 s} \bar{\theta}>\bar{v}_{0} \quad\left(y_{0 s}<0, \bar{\theta}>0\right) .
$$

For a given increase in $\bar{\theta}$ the greatest increase in the value of the solution (that is, direction of steepest ascent) is obtained by choosing $s=j$ such that

$$
\beta_{0} P_{s}=\min _{j}\left(\beta_{0} P_{j}\right)<0
$$

This establishes Theorem 3 (below) which is clearly only a restatement of the necessity of condition ( 7 ) of Theorem 1.

THEOREM 3. There exists a class of solutions with values $\bar{x}_{0}>\bar{v}_{0}$, if, for some $j=s$,

$$
y_{0 s}=\beta_{0} P_{s}<0
$$


THEOREM 4. There exists a class of solutions with no upper bound for values $\bar{x}_{0}$ if, for some $s, y_{0 s}<0$ and $y_{i s} \leq 0$ for all $i$.

THEOREM 5. There exists a new basic solution with value $\bar{x}_{0}>\bar{v}_{0}$, (obtained by introducing $P_{s}$ into the basis and dropping a unique $\left.P_{j_{r}}\right)$, if, for some $s, y_{0 s}<0$ and, for some $i, y_{i s}>0$.

Proofs. From (12), if $y_{i s} \leq 0$ for all $i$, then $\bar{\theta}$ can be arbitrarily large (that is, its first component can tend to $+\infty)$ and the coefficients of $P_{j}$ will remain nonnegative. The value of these solutions (13) will also be arbitrarily large provided that $y_{0 s}<0$ (establishing Theorem 4). In the event that some $y_{i s}>0$, the maximum value of $\bar{\theta}$ becomes

$$
\max \bar{\theta}=\left(1 / y_{r s}\right) \bar{v}_{r}=\min _{y_{i s}>0}\left(1 / y_{i s}\right) \bar{v}_{i}>0 \quad\left(y_{r s}>0, i \neq 0\right),
$$

where the minimum of the vectors (taken in the lexicographic sense) occurs for a unique $i=r$ ( since the rank of $V$ is $m+1$, no two rows of $V$ can be proportional, whereas the assumption of nonuniqueness in (16) would imply two rows of $V$ to be so - a contradiction). Setting $\bar{\theta}=\max \bar{\theta}$ in (12) yields a new basic solution since the coefficient of $P_{j_{r}}$ vanishes. Thus a new basis has been formed consisting of $\left[P_{0}, P_{j_{1}}, \cdots, P_{s}, \ldots, P_{j_{m}}\right]$, where $P_{j_{r}}$ is omitted and $P_{s}$ is put in instead ( Theorem 5 ).

The next section considers an application of the generalized simplex procedure in which the restriction $\bar{x}_{j} \geq 0$ is not imposed on all variables $(j=1$, $2, \cdots, n)$. This leads to a slight modification of procedure: first, for all $j$ for which $\bar{x}_{j} \geq 0$ is not required, both $P_{j}$ and $-P_{j}$ should be considered as columns of $P$; secondly, if $P_{j}$ is in the basis and the restriction $\bar{v}_{i}>0$ is not required, then this term cannot impose a bound on $\bar{\theta}$; hence the corresponding $i$ should be omitted from (16) in forming the minimum.

Starting with any basis $B=B^{(k)}$, one can determine a new basis $B^{(k+1)}$ by first determining the vector $P_{s}$ to introduce into the basis by (14). If there exists no $\beta_{0} P_{s}<0$, then, by Theorem 1 , the solution is optimal and $B^{(k)}$ is the final basis. If a $P_{s}$ exists, then one forms $y_{i s}=\left(\beta_{i} P_{s}\right)$ and determines the vector $P_{j_{r}}$ to drop from the basis by (16) provided that there are $y_{i s}>0$. If there exist no $y_{i s}>0$, then, by Theorem 4, a class of solutions is obtained from (12) with no upper bound for $\bar{v}_{0}$ for arbitrary $\bar{\theta}>0$. If $P_{j_{r}}$ can be determined, then a new basis $B^{(k+1)}$ is formed dropping $P_{j_{r}}$ and replacing it by $P_{s}$; 
by (13), the value, $\bar{v}_{0}$, of this solution is strictly greater for $B^{(k+1)}$ than for $B^{(k)}$ since $\bar{\theta}>0$ is chosen by (16). Thus one may proceed iteratively starting with the assumed initial basis and forming $k=0,1,2, \cdots$ until the process stops because (a) an optimal solution has been obtained, or (b) a class of solutions with no finite upper bound has been obtained.

The number of different bases is finite, not exceeding the number of combinations of $n$ things taken $m$ at a time; associated with each basis $B$ is a unique basic solution $V=B^{-1} M$ - hence the number of distinct basic solutions is finite; finally, no basis can be repeated by the iterative procedure because contrariwise this would imply a repetition of the value $\bar{v}_{0}$, whereas by (13) the values for successive basic solutions are strictly monotonically increasing-hence the number of iterations is finite.

The $(k+1)$ st iterate is closely related to the $k$ th by simple transformations that constitute the computational algorithm $[6 ; 7]$ based on the method: thus for $i=0,1, \cdots, m(i \neq r)$,

$$
\begin{array}{ll}
\bar{v}_{i}^{k+1}=\bar{v}_{i}^{k}+\eta_{i} \bar{v}_{r}^{k} ; & \bar{v}_{r}^{k+1}=\eta_{r} \bar{v}_{r}^{k} ; \\
\beta_{i}^{k+1}=\beta_{i}^{k}+\eta_{i} \beta_{r}^{k} ; & \beta_{r}^{k+1}=\eta_{r} \beta_{r}^{k},
\end{array}
$$

where the superscripts $k+1$ and $k$ are introduced here to distinguish the successive solutions and bases, and where $\eta_{i}$ are constants,

$$
\begin{array}{ll}
\eta_{i}=-y_{i s} / y_{r s}=-\left(\beta_{i} P_{s}\right) /\left(\beta_{r} P_{s}\right), & (i \neq r) \\
\eta_{r}=1 / y_{r s}=1 /\left(\beta_{r} P_{s}\right) . &
\end{array}
$$

Relation (17.0) is a consequence of (12) and (16); it is easy to verify that the matrix whose rows are defined by (17.1) satisfies the proper orthogonality properties for the inverse when multiplied on the right by the $(k+1)_{\text {st }}$ basis $\left[P_{0}, P_{j_{1}}, \cdots, P_{s}, \cdots, P_{j_{m}}\right]$. As a consequence of the iterative procedure we have established two theorems:

THEOREM 6. If solutions exist and their values have a finite upper bound, then a maximizing solution exists which is a basic solution with the properties

$$
B V=\sum_{i=0}^{m} P_{j i} \bar{v}_{i}=M
$$

$$
\left(P_{j_{0}}=P_{0}, \bar{v}_{i}>0, i=1, \cdots, m\right),
$$




$$
\begin{aligned}
\beta_{0} P_{0} & =1, \beta_{0} P_{j_{i}}=0, \beta_{0} P_{j} \geq 0 \quad(j=1,2, \cdots, n), \\
\bar{v}_{0} & =\beta_{0} M=\max \bar{x}_{0},
\end{aligned}
$$

where $\beta_{0}$ is the lst row of $B^{-1}$.

THEOREM 7. If solutions exist and their values have no finite upper bound, then a basis $B$ and a vector $P_{s}$ exist with the properties

$$
\begin{aligned}
& B V=\sum_{i=0}^{m} P_{j_{i}} \bar{v}_{i}=M \\
& \beta_{0} P_{s}<0, \beta_{i} P_{s} \leq 0, \\
& \sum P_{j_{i}}\left[\overline{v_{i}}-\left(\beta_{i} P_{s}\right) \bar{\theta}\right]+P_{s} \bar{\theta}=M,
\end{aligned}
$$

where the latter, with $\bar{\theta} \geq 0$ arbitrary, forms a class of solutions with unbounded values $\left(\beta_{i}\right.$ is the $(i+1)_{\text {st }}$ row of $\left.B^{-1}\right)$.

Closely related to the methods of the next section, a constructive proof will now be given to:

THEOREM 8. If any solution exists, then a basic solution exists.

For this purpose adjust $M$ and $P$ so that the first nonzero component of each row of $M$

$$
\begin{array}{r}
\sum_{j=0}^{n}\left[\begin{array}{l}
P_{j} \\
0
\end{array}\right] \bar{x}_{j}^{\prime}+\sum_{i=1}^{m}\left[\begin{array}{c}
U_{i} \\
1
\end{array}\right] \bar{x}_{n+i}^{\prime}+\left[\begin{array}{l}
\cdot \\
1
\end{array}\right] \bar{x}_{n+m+1}^{\prime}=\left[\begin{array}{c}
M \cdot \\
.1
\end{array}\right] \\
\left(\bar{x}_{j}^{\prime} \geq 0 ; j=1, \cdots, n+m\right),
\end{array}
$$

where $\bar{x}_{j}^{\prime}$ has one more component than $\bar{x}_{j}$, and - represents the null vector. Noting that neither $\bar{x}_{0}^{\prime}$ nor $\bar{x}_{n+m+1}^{\prime}$ is required to be positive, one sees that an obvious basic solution is obtained using the variables $\left[\bar{x}_{0}^{\prime}, \bar{x}_{n+1}^{\prime}, \ldots, \bar{x}_{n+m+1}^{\prime}\right]$. It will be noted that the hypothesis of the theorem permits construction of a solution for which

$$
\bar{x}_{n+i}^{\prime}=0
$$$$
(i=1,2, \cdots, m) \text {. }
$$

Indeed, for $j \leq n$ set $\bar{x}_{j}^{\prime}=\left(\bar{x}_{j}, 0\right)>0$. However, it will be noted also that 


$$
\sum \bar{x}_{n+i}^{\prime}=[\cdot 1]
$$

so that

$$
\max \bar{x}_{n+i}=[\cdot 1]
$$

Accordingly, one may start with the basic solution for the augmented system, keeping the vectors corresponding to $x_{0}^{\prime}$ and $x_{n+m+1}^{\prime}$ always in the basis ${ }^{1}$, and use the simplex algorithm to maximize $x_{n+m+1}^{\prime}$. Since, at the maximum,

$$
\bar{x}_{n+i}^{\prime}=0
$$

the corresponding vectors are not in the basis any longer (see (5)). By dropping the last component of this basic solution and by dropping $x_{n+m+1}^{\prime}$, one is left with a basic solution to the original system.

3. Minimizing a linear form. The application of the generalized simplex method to the problem of minimizing a linear form subject to linear inequality, constraints consists in bordering the matrix of coefficients and constant terms of the given system by appropriate vectors. This can be done in many ways - the one selected is one which identifies the inverse of the basis as the additional components in a generalized matrix problem so that computationally no additional labor is required when the inverse is known.

The fundamental problem which we wish now to solve is to find a set $x=$ $\left(x_{0}, x_{1}, \cdots, x_{n}\right)$ of real numbers satisfying the equations

$$
x_{0}+\sum_{1}^{n} a_{0 j} x_{j}=0, \sum_{1}^{n} a_{k j} x_{j}=b_{k} \quad\left(b_{k} \geq 0 ; k=2,3, \cdots, m\right),
$$

such that

$$
\begin{gathered}
x_{j} \geq 0, \\
x_{0}=\max ,
\end{gathered}
$$

where without loss of generality one may assume $b_{k} \geq 0$. It will be noted that the subscript $k=1$ has been omitted from (21). After some experimentation it has been found convenient ${ }^{2}$ to augment the equations of $(21)$ by a redundant equation formed by taking the negative sum of equations $k=2, \cdots, m$. Thus

\footnotetext{
${ }^{1}$ To accomplish this omit $i=0$ and $i=m+1$ in (16).

${ }^{2}$ Based on a recent suggestion of W. Orchard-Hays.
} 


$$
\sum_{1}^{n} a_{1 j} x_{j}=b_{1} \quad\left(a_{1 j}=-\sum_{k=2}^{m} a_{k j}, b_{1}=-\sum_{2}^{m} b_{k}\right) .
$$

Consider the generalized problem of finding a set of vector "variables" (in the sense of $\S 2)\left(\bar{x}_{0}, \bar{x}_{1}, \cdots, \bar{x}_{n}\right)$, and auxiliary variables $\left(\bar{x}_{n+1}, \bar{x}_{n+2}, \cdots\right.$, $\bar{x}_{n+m}$ ) satisfying the matrix equations

$$
\begin{aligned}
& \bar{x}_{0}+\sum_{1}^{n} a_{0 j} \bar{x}_{j}=(0,1,0, \cdots, 0), \\
& \bar{x}_{n+k}+\sum_{1}^{n} a_{k j} \bar{x}_{j}=\left(b_{k}, 0,0, \cdots, 1, \cdots, 0\right) \quad\left(b_{1} \leq 0 ; b_{k} \geq 0, k=2, \cdots, m\right),
\end{aligned}
$$

where the constant vectors have $l=m+2$ components with unity in position $k+2, \bar{x}_{0}$ and $\bar{x}_{n+1}$ are unrestricted as to sign and, for all other $j$,

$$
\bar{x}_{j} \geq 0 \quad(j=1, \cdots, n, n+2, \cdots, n+m) .
$$

Adding equations $k=1, \cdots, m$ in (25) and noting the definitions of $a_{1 j}$ and $b_{1}$ given in (24), one obtains

$$
\sum_{1}^{m} \bar{x}_{n+k}=(0,0,1,1, \cdots, 1)
$$

There is a close relationship between the solutions of (25) and those of (21) when $\bar{x}_{n+1} \geq 0$, for then the first components of $\bar{x}_{j}$, for $j=0, \cdots, n$, satisfy (21). Indeed, by (27), if all $\bar{x}_{n+k} \geq 0$, the first component of all $\bar{x}_{n+k}$ must vanish; but the first component of the vector equations (25) reduces to (21) when the terms involving $x_{n+k}$ are dropped. This proves the sufficiency of Theorem 9 (below).

THEOREM 9. A necessary and sufficient condition for a solution of (21) to exist is for a solution of (25) to exist with $\bar{x}_{n+1} \geq 0$.

THEOREM 10. Maximizing solutions (or a class of solutions with unbounded values) of (21) are obtained from the lst components of $\left(\bar{x}_{0}, \cdots, \bar{x}_{n}\right)$ of the corresponding type solution of (25) with $\bar{x}_{n+1} \geq 0$.

Proofs. To prove necessity in Theorem 9, assume $\left(x_{0}, \cdots, x_{n}\right)$ satisfies 
(21); then the set

$$
\begin{array}{rlrl}
\bar{x}_{0} & =\left(x_{0}, 1,0, \cdots, 0\right), \\
\bar{x}_{j} & =\left(x_{j}, 0,0, \cdots, 0\right) & & (1 \leq j \leq n), \\
\bar{x}_{n+k} & =(0,0, \cdots, 1, \cdots, 0) \geq 0 & (1 \leq k \leq m)
\end{array}
$$

(where unity occurs in position $k+2$ ) satisfies (25). Because of the possibility of forming solutions of the type (28) from solutions of (21), it is easy to show that lst components of maximizing solutions of (25) must be maximizing solutions of (28) (Theorem 10).

It will be noted that (25) satisfies the requirements for the generalized simplex process: first the right side considered as a matrix is of the form

$$
M=\left[Q, U_{0}, U_{1}, \cdots, U_{m}\right]
$$

where $U_{k}$ is a unit column vector with unity in component $k+1$, and is of rank $m+1$ (the number of equations); second, an initial basic solution is available. Indeed, set $\bar{x}_{0}, \bar{x}_{n+1}, \bar{x}_{n+2}, \cdots, \bar{x}_{n+m}$ equal to the corresponding constant vectors in (25) where $\bar{x}_{n+k} \geq 0$ for $k=2, \cdots, m$ because $b_{k} \geq 0$.

In applying the generalized simplex procedure, however, both $\bar{x}_{0}$ and $\bar{x}_{n+1}$ are not restricted to be nonnegative. Since $\bar{x}_{n+k} \geq 0$ for $k=2, \cdots, m$, it follows that the values of the solutions, $\bar{x}_{n+1}$, of (27) have the right side of (27) as an upper bound.

To obtain a maximizing solution of (25), the first phase is to apply the generalized simplex procedure to maximize the variable $\bar{x}_{n+1}$ (with no restriction on $\bar{x}_{0}$ ). Since $\bar{x}_{n+1}$ has a finite upper bound, a basic solution will be produced after a finite number of changes of basis in which $\bar{x}_{n+1} \geq 0$, provided that $\max \bar{x}_{n+1} \geq 0$. If during the first phase $x_{n+1}$ reaches a maximum less than zero, then, of course, by Theorem 9 there is no solution of (21) and the process terminates. If, in the iterative process, $\bar{x}_{n}+1$ becomes positive (even though not maximum), the first phase, which is the search for a solution of (21), is completed and the second phase, which is the search for an optimal solution, begins. Using the final basis of the first phase in the second phase, one sees that $\bar{x}_{0}$ is maximized under the additional constraint $\bar{x}_{n+1} \geq 0$.

Since the basic set of variables is taken in the initial order $\left(\bar{x}_{0}, \bar{x}_{n}+1, \cdots\right.$, $\left.\bar{x}_{n+m}\right)$, and in the first phase the variable $\bar{x}_{n+1}$ is maximized, the second row 
of the inverse of the basis, $\beta_{1}$, is used to "select" the candidate $P_{s}$ to introduce into the basis in order to increase $\bar{x}_{n+1}$ (see (14)); hence $s$ is determined such that

$$
\beta_{1} P_{s}=\min _{j}\left(\beta_{1} P_{j}\right)<0
$$

However, in the second phase, since the variable to be maximized is $\overline{x_{0}}$ and the order of the basic set of variables is $\left(\bar{x}_{0}, \bar{x}_{n+1}, \ldots\right)$, then the first row of the inverse of the basis, $\beta_{0}$, is used; that is, one reverts back to (14). Application of the generalized simplex procedure in the second phase yields, after a finite number of changes in basis, either a solution with max $\bar{x}_{0}$ or a class of solutions of form (12) with no upper bound for $\bar{x}_{0}$. By Theorem 10 the first components of $\bar{x}_{0}, \bar{x}_{1}, \ldots, \bar{x}_{n}$ form the corresponding solutions of the real variable problem.

The computational convenience of this setup is apparent. In the first place (as noted earlier), the right side of (21) considered as a matrix is of the form

$$
M=\left[Q, U_{0}, U_{1}, \cdots, U_{m}\right],
$$

where $U_{i}$ is a unit column vector with unity in component $k+1$. In this case, by (4), the basic solution satisfies

$$
V=B^{-1} M=\left[B^{-1} Q ; B^{-1}\right]
$$

This means (in this case) that of the $l=m+2$ components of the vector $\vec{v}_{i}$ the last $m+1$ components of the vector variables $\bar{v}_{i}$ in the basic solution are identical with $\beta_{i}$, the corresponding row of the inverse. In applications this fact is important because the last $m+1$ components of $\bar{v}_{i}$ are artificial in the sense that they belong to the perturbation and not to the original problem and it is desirable to obtain them with as little effort as possible. In the event that $M$ has the foregoing special form, no additional computational effort is required when the inverse of the basis is known. Moreover, the columns of (25) corresponding to the $m+1$ variables $\left(\bar{x}_{0}, \bar{x}_{m+1}, \cdots, \bar{x}_{n+m}\right)$ form the initial identity basis $\left(U_{0}, U_{1}, \cdots, U_{m}\right)$, so that the inverse of the initial basis is readily available as the identity matrix to initiate the first iteration.

\section{REFERENCES}

1. Activity analysis of production and allocation, T.C. Koopmans, Editor, John Wiley and Sons, New York, 1951. 
(a) R. Dorfman, Application of simplex method to a game theory problem, Chapter XXII.

(b) G. Dantzig, Maximization of a linear function of variables subject to linear inequalities, Chapter XXI.

(c) G. Dantzig, Application of the simplex method to a transportation problem, Chapter XXIII.

(d) D. Gale, H. Kuhn, and A. Tucker, Linear programming and the the ory of games, Chapter XIX.

2. A. Charnes, Optimality and degeneracy in linear programming, Econometrica, April 1952, pages 160-70.

3. A. Charnes, W. W. Cooper, and A. Henderson, An introduction to linear programming, John Wiley and Sons, New York, 1953.

4. A. Charnes and C. E. Lemke, Computational problems of linear programming, Proceeding of the Association of Computation Machinery, Pittsburgh, 1952, pages 97-98.

5. Contributions to Theory of Games, H. Kuhn and A. W. Tucker, Editors, Princeton University Press; Vol. I, 1950; Vol. II, 1953.

6. G. Dantzig, Computational Algorithm of the Revised Simplex Method, RAND P-394-1, October 1953.

7. G. Dantzig and W. Orchard-Hays, The product form for the inverse in the simplex method, RAND P-394-1, October 1953; also RAND P-460, November 1953.

8. G. Dantzig and A. Orden, A duality theorem based on the simplex method, Symposium on Linear Inequalities, USAF-Hq., SCOOP Publication No. 10, dated 1 April 1952, pages $51-55$.

9. A. Hoffman, M. Mannos, D. Sokolowsky, and N. Wiegmann, Computational experience in solving linear programs, Journal of Social Industrial and Applied Mathematics, Vol. 1, No. 1, September 1953, pages 17-33.

10. T.S. Motzkin, Two consequences of the transposition theorem of linear inequalities, Econometrica, Vol. 19, No. 2, April 1951, pages 184-5.

11. Beiträge zur Theorie der Linearen Ungleichungen, Dissertation, Basel, 1933; Jerusalem, 1935.

12. J. von Neumann, Discussion of a maximization problem, Institute for Advanced Study, 1947 Manuscript.

13. Alex Orden, Solution of systems of linear inequalities on a digital computer, Proceedings of the Association of Computing Machinery, Pittsburgh, 1952, pages 91-95.

14. A. J. Hoffman, Cycling in the simplex algorithm, National Bureau of Standards Report, No. 2974, December 16, 1953.

\section{RAND CORPORATION}

BURR OUGHS CORPORATION

PRINCETON UNIVERSITY 



\section{PACIFIC JOURNAL OF MATHEMATICS}

\section{EDITORS}

\author{
H. L. ROYDEN \\ Stanford University \\ Stanford, California \\ E. Hewitt \\ University of Washington \\ Seattle 5 , Washington
}

R. P. Dilworth

California Institute of Technology Pasadena 4, California

A. HorN*

University of California

Los Angeles 24, California

\section{ASSOCIATE EDITORS}

\author{
H. BUSEMANN \\ HERBERT FEDERER \\ MARSHALL HALL
}

\author{
P. R. HALMOS \\ HEINZ HOPF
}

ALFRED HORN
R. D. JAMES

BORGE JESSEN

PAUL LÉVY
GEORGE PÓLYA

J. J. STOKER

KOSAKU YOSIDA

\section{SPONSORS}

UNIVERSITY OF BRITISH COLUMBIA

CALIFORNIA INSTITUTE OF TECHNOLOGY

UNIVERSITY OF CALIFORNIA, BERKELEY

UNIVERSITY OF CALIFORNIA, DAVIS

UNIVERSITY OF CALIFORNIA, LOS ANGELES

UNIVERSITY OF CALIFORNIA, SANTA BARBARA

MONTANA STATE UNIVERSITY

UNIVERSITY OF NEVADA

OREGON STATE COLLEGE

UNIVERSITY OF OREGON

UNIVERSITY OF SOUTHERN CALIFORNIA

\author{
STANFORD RESEARCH INSTITUTE \\ STANFORD UNIVERSITY \\ UNIVERSITY OF UTAH \\ WASHINGTON STATE COLLEGE \\ UNIVERSITY OF WASHINGTON
}

AMERICAN MATHEMATICAL SOCIETY HUGHES AIRCRAFT COMPANY SHELL DEVELOPMENT COMPANY

Mathematical papers intended for publication in the Pacific Journal of Mathematics should be typewritten (double spaced), and the author should keep a complete copy. Manuscripts may be sent to any of the editors. Manuscripts intended for the outgoing editors should be sent to their successors. All other communications to the editors should be addressed to the managing editor, Alfred Horn at the University of California, Los Angeles 24, California.

50 reprints of each article are furnished free of charge; additional copies may be obtained at cost in multiples of 50 .

The Pacific Journal of Mathematics is published quarterly, in March, June, September, and December. The price per volume (4 numbers) is $\$ 12.00$; single issues, $\$ 3.50$. Back numbers are available. Special price to individual faculty members of supporting institutions and to individual members of the American Mathematical Society: $\$ 4.00$ per volume; single issues, $\$ 1.25$.

Subscriptions, orders for back numbers, and changes of address should be sent to Pacific Journal of Mathematics, c/o University of California Press, Berkeley 4, California.

Printed at Kokusai Bunken Insatsusha (International Academic Printing Co., Ltd.), No. 10, 1-chome, Fujimi-cho, Chiyoda-ku, Tokyo, Japan.

* During the absence of E. G. Straus.

PUBLISHED BY PACIFIC JOURNAL OF MATHEMATICS, A NON-PROFIT CORPORATION COPYRIGHT 1955 BY PACIFIC JOURNAL OF MATHEMATICS 


\section{Pacific Journal of Mathematics}

\section{Vol. 5, No. $2 \quad$ October, 1955}

Leonard M. Blumenthal, An extension of a theorem of Jordan and von

Neumann ........................................ 161

L. Carlitz, Note on the multiplication formulas for the Jacobi elliptic functions.......................................... 169

L. Carlitz, The number of solutions of certain types of equations in a finite

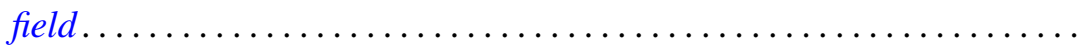

George Bernard Dantzig, Alexander Orden and Philip Wolfe, The generalized simplex method for minimizing a linear form under linear

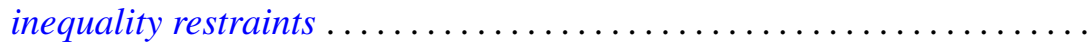

Arthur Pentland Dempster and Seymour Schuster, Constructions for poles and polars in n-dimensions . . . . . . . . . . . . . . . . . . . . 197

Franklin Haimo, Power-type endomorphisms of some class 2 groups ...... 201

Lloyd Kenneth Jackson, On generalized subharmonic functions ......... 215

Samuel Karlin, On the renewal equation ...................... 229

Frank R. Olson, Some determinants involving Bernoulli and Euler numbers of higher order................................ 259

R. S. Phillips, The adjoint semi-group ........................ 269

Alfred Tarski, A lattice-theoretical fixpoint theorem and its applications ... 285

Anne C. Davis, A characterization of complete lattices .............. 311 